\title{
SHIP WAKE COMPONENT DETECTABILITY ON SYNTHETIC APERTURE RADAR (SAR)
}

\author{
Björn Tings, Stefan Wiehle, Sven Jacobsen \\ German Aerospace Center (DLR), Am Fallturm 9, 28359 Bremen, Germany
}

\begin{abstract}
This study presents an extension to recent ship wake detectability models based on machine learning by modelling the detectability of certain wake components individually. Our recent studies investigated the detectability of ship wake signatures on SAR imagery by collocation with Automatic Identification System data. The developed detectability models are based on machine learning and coincide with findings from previous research on wake detectability using simulations or qualitative image analysis. Additionally, this previous research also provides information on the detectability of individual wake components, which is qualitatively investigated in this study using a set of training data extended by manually labelled wake components.
\end{abstract}

Index Terms - Wake Detectability, Wake Component, Object Detection, Machine Learning, Synthetic Aperture Radar, five

\section{INTRODUCTION}

The information content of current SAR ship detection systems can be increased by taking the ship wake signatures into account, which are visible behind vessels with a sufficiently high travelling speed [1]. Previous researchers already explained the visibility of ship wakes by applying knowledge of the underlying physics to perform qualitative image analysis as well as simulation models. The detectability of such ship wakes and individual wake components depends on various influencing parameters, which can be assigned to the following three categories: environmental conditions, ship properties and image acquisition settings [2]. Our previous studies show that the dependency of wake detectability on the influencing parameters can be modelled by ma-chine learning (ML) methods $[3,4]$. The resulting models basically coincide with the oceanographic expectations defined by previous researchers through simulation or qualitative image analysis $[3,4]$.

However, in [3] and [4] the detectability of wakes was investigated without differentiation between the individual wake components: turbulent wake, Kelvin wakes, V-Narrow wakes and internal waves [5]. Especially in [4] some of the results could only be explained by the different imaging mechanisms of these wake components. A quantitative investigation of the detectability of ship wake components using real data as basis is therefore pending and provided by this study.

The next section updates the results from [3] and [4], while section 3 presents the initial results obtained by the new individual models for certain wake components. The study is concluded at the end of the paper.

\section{ML-BASED DETECTABILITY MODELS}

The models presented in [3] and [4] are updated using a larger training dataset. The creation of the ML-based models is briefly described in the following subsections. More details can be gathered from the respective publications.

\subsection{Linear wake detectability models}

In case only few SAR scenes with Automatic Identification System (AIS) information are available, the detectability should be modelled using a linear basis. This means only the influencing parameters, which showed a linear (i.e. independent monotonic influence) on the wake detectability in [4] can be considered.

The creation of the linear detectability models can be summarized by the following steps:

1. Localizing moving vessels by AIS data collocated with SAR images

2. Extracting training dataset consisting of wake samples at identified locations

3. Manually inspecting the wake samples with subsequent labelling by class labels "detected" or "not detected"

4. Extracting influencing parameters: Inci-denceAngle, AIS-Vessel-Velocity, AIS-Ship-Length and SARWind-Speed

5. Training logistic regression classifiers for discrimination of two classes using selected subsets of influencing parameters

6. Quantification of detectability for each parameter subset by probability of class affiliation to class "detected"

By sampling the whole feature space of each influencing parameter in the parameter subsets and feeding these artificial data points into the linear classification model, the 
nature of the model can be visualized. The resulting 2Ddetectablity charts can be used to derive information about the detectability of ship wakes in dependency to the respective influencing parameters. An example 2Ddetectablity chart is presented by Figure 1 .

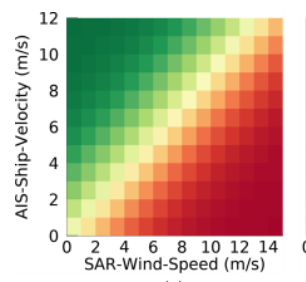

(a)

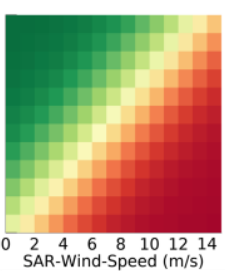

(b)

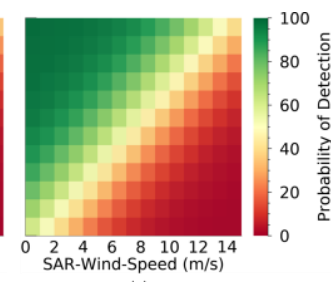

(c)
Figure 1 TerraSAR-X high-resolution 2D-detectability charts based on SAR-Wind-Speed, AIS-Ship-Velocity and from left to right $25 \mathrm{~m}, 50 \mathrm{~m}$, and $100 \mathrm{~m}$ SAR-Ship-Length.

\subsection{Non-linear wake detectability models}

The amount of TerraSAR-X data available for this study was sufficient for training a non-linear wake detectability model. Thus, influencing parameters with non-linear influence on wake detectability can be considered. With a sufficient amount of data, influencing parameters can be combined into one model instead of selecting parameter subsets. Then, also interdependencies between the parameters can be represented by the model.

Two of the previous steps are changed to obtain a non-linear detectability model:

4. Extracting four additional influencing pa-rameters: AIS-CoG, SAR-Significant-Wave-Height, AIS- CoG-SARWave-Direction

5. Training of one Support Vector Machine (SVM) classifier with polynomial kernel of degree two

Identical to the linear detectability model, 2Ddetectability charts can be derived for model visualization. In Figure 2 an example 2D-detectablity chart is presented, which presents the non-linear nature of the underlying model and its capability to depict interdependencies.

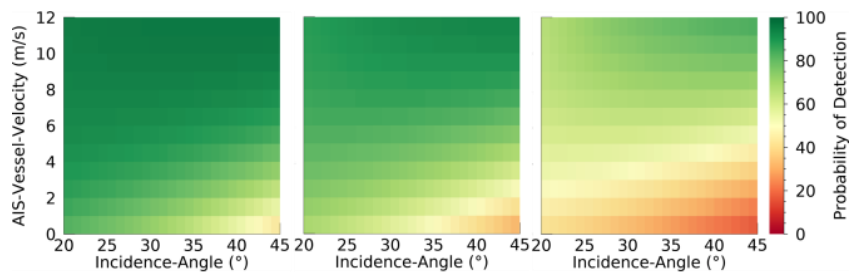

(a)

(b)

(c)

Figure 2 2D-detectability charts based on Incidence-Angle, AIS-Vessel-Velocity and from left to right $1 \mathrm{bft}, 3 \mathrm{bft}$ and 5 bft Beaufort numbers.

\section{WAKE COMPONENT DETECTABILITY}

The former approach described in Section 2.2 and with more detail in [4] is adopted in this study for the modelling of detectability of individual wake components. All wake samples in the training dataset went through additional manual inspection, where all four wake component types (turbulent wake, Kelvin wakes, V-narrow wakes and internal waves) were delineated. The resulting dataset now also contains information about the position, orientation and length of the individual wake components. This means a detectability model can now be trained for each component type. Further, the underlying ML-method can exploit the additional scalar information created by the manual inspection, which enables the training of a regression model instead of a binary classifier.

As an initial approach, the delineated component length is used as outcome variable of a Support Vector Regression (SRV) model. Comparable hyperparameter settings and an identical list of influencing parameters (i.e. features) were used as specified for the SVM model used in Section 2.2. The model is still under preparation, but already some consistent 2D-detectablity charts can be derived for some of the wake component types and for a subset of influencing parameters.

Example 2D-detectability charts show the initial results in Figure 3 and Figure 4. They provide information about the detectability of the turbulent wake and the Kelvin wake in dependency to the influencing parameters, which were also used in Figure 2.

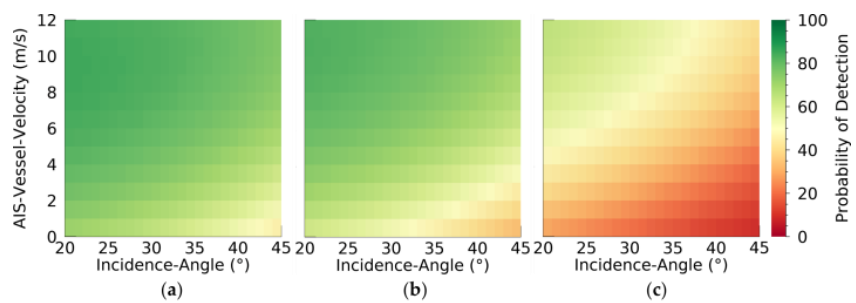

Figure $32 \mathrm{D}$-detectability charts for turbulent wake based on Incidence-Angle, AIS-Vessel-Velocity and from left to right $1 \mathrm{bft}, 3 \mathrm{bft}$ and $5 \mathrm{bft}$ Beaufort numbers.

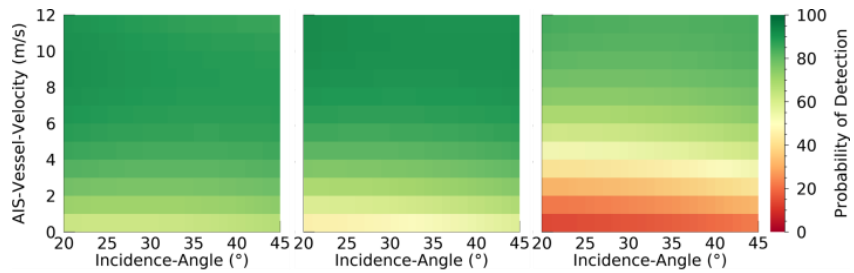

(a)

(b)

(c)

Figure 4 2D-detectability charts for Kelvin wake based on Incidence-Angle, AIS-Vessel-Velocity and from left to right $1 \mathrm{bft}, 3 \mathrm{bft}$ and $5 \mathrm{bft}$ Beaufort numbers.

The turbulent wake and the Kelvin wake are the two wake component types, which frequently appear in SAR imagery. Therefore, in [4] it was already presumed that the 
detectability model for full wake signatures would mainly be based on the detectability of these two wake component types. Now, by comparison of Figures 3 and 4 with Figure 2, this statement is supported, because Figure 2 can be interpreted as being a composite of Figures 3 and 4 .

In [4] and by previous researchers was already explained, why turbulent wakes are better detectable under lower incidence angles and Kelvin wakes are better detectable under higher incidence angles. The initial results with the wake component detectability model support these expectations.

\section{CONCLUSION}

In this study a method for modelling the detectability of individual wake components in dependency to influencing parameters is presented. The method is an extension to a previous approach for modelling the detectability of full wake signatures in dependency to the influencing parameters [4].

Initial results demonstrate the ability of the presented approach to model the detectability of individual wake components in agreement with oceanographic expectations. One statement made in [4] about the dependency of wake's detectability on the influencing parameter "incidence angle" can hereby be confirmed.

\section{REFERENCES}

[1] M. D. Graziano, A. Renga, and A. Moccia, "Integration of Automatic Identification System (AIS) Data and SingleChannel Synthetic Aperture Radar (SAR) Images by SARbased Ship Velocity Estimation for Maritime Situational Awareness," Remote Sens., vol. 11, no. 19, pp. 1-16, Sept. 2019

[2] I. Hennings, R. Romeiser, W. Alpers, and A. Viola, "Radar imaging of Kelvin arms of ship wakes," Int. J. Remote Sens., vol. 20, no. 13, pp. 2519-2543, 1999

[3] B. Tings, A. Pleskachevsky, D. Velotto, and S. Jacobsen, "Extension of Ship Wake Detectability Model for NonLinear Influences of Parameters Using Satellite Based XBand Synthetic Aperture Radar," Remote Sens., vol. 11, no. 5, pp. 1-20, Mar. 2019

[4] B. Tings and D. Velotto "Comparison of ship wake detectability on C-band and X-band SAR," Int. J. Remote Sens., vol. 39, no. 13, pp. 4451-4468, Jan. 2018

[5] J.D. Lyden, R.R. Hammond, D.R. Lyzenga, and R. Shuchman, "Synthetic Aperture Radar Imaging of Surface Ship Wakes," J. Geophys. Res., vol 93, no. C10, pp. 1229312303, Oct. 1988 